\title{
Double product integrals and Enriquez quantisation of Lie bialgebras II: The quantum Yang-Baxter equation.
}

\author{
R L Hudson †and S Pulmannováł \\ Received 30 September 2003, revised 21 April 2005
}

\begin{abstract}
For a Lie algebra with Lie bracket got by taking commutators in a nonunital associative algebra $\mathscr{L}$, let $\mathscr{T}(\mathscr{L})$ be the vector space of tensors over $\mathscr{L}$ equipped with the Itô Hopf algebra structure derived from the associative multiplication in $\mathscr{L}$. It is shown that a necessary and sufficient condition that the double product integral $\vec{\prod}(1+d r[h])$ satisfy the quantum Yang-Baxter equation over $\mathscr{T}(\mathscr{L})$ is that $1+r[h]$ satisfy the same equation over the unital associative algebra $\mathscr{L}^{\prime}$ got by adjoining a unit element to $\mathscr{L}$. In particular the first-order coefficient $r_{1}$ of $r[h]$ satisfies the classical Yang-Baxter equation. Using the fact that the multiplicative inverse of $\vec{\prod}(1+d r[h])$ is $\overleftrightarrow{\Pi}\left(1+d r^{\prime}[h]\right)$ where $1+r^{\prime}[h]$ is the inverse of $1+r[h]$ in $\mathscr{L}^{\prime}[[h]]$ we construct a quantisation of an arbitrary quasitriangular Lie bialgebra structure on $\mathscr{L}$ in the unital associative subalgebra of $\mathscr{T}(\mathscr{L})[[h]]$ consisting of formal power series whose zero order coefficient lies in the space $\mathscr{S}(\mathscr{L})$ of symmetric tensors. The deformation coproduct acts on $\mathscr{S}(\mathscr{L})$ by conjugating the undeformed coproduct by $\vec{\prod}(1+d r[h])$ and the coboundary structure $r$ of $\mathscr{L}$ is given by $r=r_{1}-\tau_{(2,1)} r_{1}$ where $\tau_{(2,1)}$ is the flip.
\end{abstract}

\section{Introduction.}

Let $\mathscr{L}$ be a finite dimensional Lie algebra in which the Lie bracket is got by taking commutators in a not necessarily unital associative algebra $\mathscr{L}$. In a previous article

\footnotetext{
*AMS Classification 53D55, 17B62; keywords: quantisation, Lie bialgebras, quantum Yang-Baxter equation

${ }^{\dagger}$ School of Computing and Mathematics, Nottingham Trent University, Burton Street, Nottingham NG1 4BU, UK. robin.hudson@ntu.ac. uk

${ }^{\ddagger}$ Mathematical Institute, Slovak Academy of Sciences, Stefanikova 49, 81473 Bratislava, Slovakia. pulmann@mat.savba.sk
} 
[6] (see also [2]) we showed that, in the Hopf algebra $\mathscr{T}(\mathscr{L})[[h]]$ of formal power series with coefficients in the space $\mathscr{T}(\mathscr{L})$ of all tensors over $\mathscr{L}$ equipped with the noncommutative Itô extension of the shuffle product, nonzero elements $R[h]$ of $(\mathscr{T}(\mathscr{L}) \otimes \mathscr{T}(\mathscr{L}))[[h]]$ satisfying the quasitriangularity relations

$$
(\Delta \otimes \operatorname{id}(\mathscr{T})) R[h]=R^{1,3}[h] R^{2,3}[h], \quad(\operatorname{id} \mathscr{T}(\mathscr{L}) \otimes \Delta) R[h]=R^{1,3}[h] R^{1,2}[h]
$$

in the convolution algebra $(\mathscr{T}(\mathscr{L}) \otimes \mathscr{T}(\mathscr{L}) \otimes \mathscr{T}(\mathscr{L}))[[h]]$, where $\Delta$ is the coprod-

uct, can be characterised as directed double product integrals of form $\vec{\prod}(1+d r[h])$, where $r[h]$ is an element of the space $h(\mathscr{L} \otimes \mathscr{L})[[h]]$ of formal power series with coefficients in $\mathscr{L} \otimes \mathscr{L}$ and vanishing constant term.

In the present article, we shall first establish the following necessary and sufficient condition on $r[h]$ for such a product integral $R[h]=\vec{\prod}(1+d r[h])$ to satisfy the quantum Yang-Baxter equation

$$
R^{1,2}[h] R^{1,3}[h] R^{2,3}[h]=R^{2,3}[h] R^{1,3}[h] R^{1,2}[h]
$$

in the algebra of formal power series $(\mathscr{T}(\mathscr{L}) \otimes \mathscr{T}(\mathscr{L}) \otimes \mathscr{T}(\mathscr{L}))[[h]]$.

Theorem 1 A necessary and sufficient condition that the product integral $\stackrel{\leftarrow}{\prod}(1+$ $d r[h])$ satisfy the quantum Yang-Baxter equation (2) is that

$$
\begin{aligned}
& r^{1,2}[h] r^{1,3}[h]+r^{1,2}[h] r^{2,3}[h]+r^{1,3}[h] r^{2,3}[h]+r^{1,2}[h] r^{1,3}[h] r^{2,3}[h] \\
= & r^{1,3}[h] r^{1,2}[h]+r^{2,3}[h] r^{1,2}[h]+r^{2,3}[h] r^{1,3}[h]+r^{2,3}[h] r^{1,3}[h] r^{1,2}[h] .
\end{aligned}
$$

in the associative algebra $(\mathscr{L} \otimes \mathscr{L} \otimes \mathscr{L})[[h]]$.

If $\mathscr{L}^{\prime}$ is the algebra got by appending a unit element to $\mathscr{L}$ we see by adding the appropriate unit element to both sides that (3) is equivalent to the condition that $1+r[h]$ satisfies the quantum Yang Baxter equation in $\left(\mathscr{L}^{\prime} \otimes \mathscr{L}^{\prime} \otimes \mathscr{L}^{\prime}\right)[[h]]$,

$$
\left(1+r^{1,2}[h]\right)\left(1+r^{1,3}[h]\right)\left(1+r^{2,3}[h]\right)=\left(1+r^{2,3}[h]\right)\left(1+r^{1,3}[h]\right)\left(1+r^{1,2}[h]\right) .
$$

In [7] it was claimed erroneously that the symmetrised double product integral $\Pi \prod(1+d r[h])$ satisfied the quantum Yang-Baxter equation if $r[h]$ satisfies (3); in fact for symmetrised integrals (3) is sufficient for the quantum Yang-Baxter equation to hold only to order $h^{3}$.

The condition (3) also occurs in a somewhat different context in [1]. Equating coefficients of $h^{2}, h^{3}, \ldots$ in (3) we find firstly that the first order coefficient $r_{1} \in$ $\mathscr{L} \otimes \mathscr{L}$ of $r$ satisfies the classical Yang-Baxter equation

$$
\left[r_{1}^{1,2}, r_{1}^{1,3}\right]+\left[r_{1}^{1,2}, r_{1}^{2,3}\right]+\left[r_{1}^{1,3}, r_{1}^{2,3}\right]=0,
$$


while for higher order coefficents

$$
\begin{array}{r}
\sum_{s+t=N+1}\left(\left[r_{s}^{1,2}, r_{t}^{1,3}\right]+\left[r_{s}^{1,2}, r_{t}^{2,3}\right]+\left[r_{s}^{1,3}, r_{t}^{2,3}\right]\right) \\
+\quad \sum_{s+t+u=N+1}\left(r_{s}^{1,2} r_{t}^{1,3} r_{u}^{2,3}-r_{u}^{2,3} r_{t}^{1,3} r_{s}^{1,2}\right)=0
\end{array}
$$

where the sums are over ordered pairs $(s, t)$ and triples $(s, t, u)$ of natural numbers whose sums are $N+1$. Assuming that $r_{1}, r_{2}, \ldots, r_{N-1}$ have been found, (6) gives a finite dimensional inhomogeneous linear equation for $r_{N}$. It is shown in [1] that, for a given solution $r_{1}$ of (5), the resulting hierarchy of equations has solutions, however, to make this inference, the existence of a quantisation of an arbitrary Lie bialgebra, as proved in [3], is needed.

Our second Theorem is that the directed double product integral $\stackrel{\leftarrow}{\prod}(1+d r[h])$ has a multiplicative inverse which is also a directed product integral but with arrows reversed.

Theorem 2 For arbitrary $r[h] \in h(\mathscr{L} \otimes \mathscr{L})[[h]], \stackrel{+\leftarrow}{\prod}(1+d r[h])$ is invertible in $(\mathscr{T}(\mathscr{L}) \otimes \mathscr{T}(\mathscr{L}))[[h]]$ with inverse $\stackrel{\leftarrow}{\Pi}\left(1+d r^{\prime}[h]\right)$ where $r^{\prime}[h]$ is the quasiinverse of $r[h]$ in $h \mathscr{L}[[h]]$, that is, the unique element satisfying

$$
r[h]+r^{\prime}[h]+r[h] r^{\prime}[h]=r^{\prime}[h]+r[h]+r^{\prime}[h] r[h]=0 .
$$

Thus $1+r^{\prime}[h]$ is the inverse of $1+r[h]$ in $\left(\mathscr{L}^{\prime} \otimes \mathscr{L}^{\prime}\right)[[h]]$.

Let us now demonstrate how Theorems 1 and 2 permit a simple construction of a deformed Hopf algebra, which as an associative algebra is a subalgebra of the Itô shuffle algebra $\mathscr{T}(\mathscr{L})[[h]]$, whose semi-classical limit is an arbitrary quasitriangular Lie bialgebra with commutator Lie bracket. This may be compared with the general construction of Enriquez [1].

We first recall [5] that the subspace $\mathscr{S}(\mathscr{L})$ of $\mathscr{T}(\mathscr{L})$ consisting of symmetric tensors is isomorphic as a Hopf algebra to the universal enveloping algebra $\mathscr{U}(\mathscr{L})$ of the Lie algebra $\mathscr{L}$. Let us now consider the algebra $\mathscr{T}(\mathscr{L})_{0}[[h]]$ consisting of formal power series with coefficients in $\mathscr{T}(\mathscr{L})$ whose zero-order coefficients are in $\mathscr{S}(\mathscr{L})$. We define a deformed coproduct $\Delta[h]$ on $\mathscr{T}(\mathscr{L})_{0}[[h]]$ by

$$
\Delta[h](\alpha)=R[h] \Delta(\alpha) R[h]^{-1}
$$

where $R[h]=\overleftrightarrow{\prod^{\leftarrow}}(1+d r[h])$, and consequently also its inverse $R[h]^{-1}=\overleftrightarrow{\Pi}(1+$ $\left.d r^{\prime}[h]\right)$, satisfy the quantum Yang-Baxter equation (2). Using (2) together with the quasitriangular relations (11) and the reversed quasitriangular relations

$$
\left(\Delta \otimes \operatorname{id}_{\mathscr{T}(\mathscr{L})}\right) R[h]^{-1}=R^{2,3}[h]^{-1} R^{1,3}[h]^{-1},\left(\operatorname{id}_{\mathscr{T}(\mathscr{L})} \otimes \Delta\right) R[h]^{-1}=R^{1,2}[h]^{-1} R^{1,3}[h]^{-1}
$$


satisfied by $R[h]^{-1}$ and the multiplicativity and coassociativity of the undeformed coproduct $\Delta$, we have

$$
\begin{aligned}
& \left(\Delta[h] \otimes \operatorname{id}_{\mathscr{T}(\mathscr{L})}\right) \Delta[h](\alpha) \\
= & R^{1,2}[h] R^{1,3}[h] R^{2,3}[h]\left(\Delta \otimes \operatorname{id}_{\mathscr{T}(\mathscr{L})}\right) \Delta(\alpha) R^{2,3}[h]^{-1} R^{1,3}[h]^{-1} R^{1,2}[h]^{-1} \\
= & R^{2,3}[h] R^{1,3}[h] R^{1,2}[h](\operatorname{id} \mathscr{T}(\mathscr{L}) \otimes \Delta) \Delta(\alpha) R^{1,2}[h]^{-1} R^{1,3}[h]^{-1} R^{2,3}[h]^{-1} \\
= & (\operatorname{id} \mathscr{T}(\mathscr{L}) \otimes \Delta[h]) \Delta[h](\alpha)
\end{aligned}
$$

so that $\Delta[h]$ is coassociative.

The Lie bialgebra cobracket of the semiclassical limit of this deformation is given [4] by

$$
\delta(L)=h^{-1}\left(\Delta[h](L)-\Delta[h]^{\mathrm{opp}}(L)\right)+o(h), L \in \mathscr{L}
$$

Using the facts that

$$
\vec{\prod}(1+d r[h])=1_{\mathscr{T}(\mathscr{L})}+r[h]+o\left(h^{2}\right), \stackrel{\overleftarrow{\prod}}{\prod^{\prime}}\left(1+d r^{\prime}[h]\right)=1_{\mathscr{T}(\mathscr{L})}+r^{\prime}[h]+o\left(h^{2}\right)
$$

and $\left(r^{\prime}\right)_{1}=-r_{1}$ we find that

$$
\delta(L)=\left[r_{1}-\tau_{(2,1)} r_{1}, L^{1}+L^{2}\right] .
$$

Since $r_{1}$ satisfies the classical Yang-Baxter equation, the Lie bialgebra $(\mathscr{L},[.,],. \delta)$ is quasitriangular, and every commutator quasitriangular Lie bialgebra can be quantised in this way.

In Section 2 we review the theory of ordered double product integrals. Some properties of the coproduct are reviewed in Section 3 and used to derive another necessary and sufficient condition that a product integral satisfy the quantum YangBaxter equation. In Section 4 we show that this second condition reduces to (3) and thus complete the proof of Theorem 1. In Section 5 we prove Theorem 2. In section 6 we describe an example of our quantisation procedure in detail.

Acknowledgement. The authors wish to thank the referees, and especially Benjamin Enriquez, for suggestions and criticisms which have greatly improved the first draft of this paper.

\section{Ordered double product integrals.}

For $r[h] \in h(\mathscr{L} \otimes \mathscr{L})[[h]]$ the ordered double products $\stackrel{\leftarrow}{\Pi}(1+d r[h]), \overleftarrow{\Pi}(1+$ $\left.d r^{\prime}[h]\right)$ are defined [6] by

$$
\begin{aligned}
& \vec{\prod}(1+d r[h])=\mathscr{\leftarrow}(\mathscr{L}) \vec{\prod}\left(1+d\left(\hat{\prod}_{\mathscr{L}}(1+d r[h])\right)=\overleftarrow{\prod} \mathscr{T}(\mathscr{L})\left(1+d\left(\mathscr{L} \hat{\vec{\prod}}(1+d r[h])\right),\right.\right. \\
& \overleftarrow{\prod}\left(1+d r^{\prime}[h]\right)=\mathscr{T}_{\mathscr{L}} \overleftarrow{\prod}\left(1+d\left(\hat{\prod}_{\mathscr{L}}(1+d r[h])\right)=\vec{\prod}_{\mathscr{T}(\mathscr{L})}\left(1+d\left(\mathscr{L} \hat{\prod}^{(1+d r[h]))}\right.\right.\right.
\end{aligned}
$$


Here the notations are as follows. Given a unital associative algebra $\mathscr{A}$ and an element $l[h]$ of $h(\mathscr{A} \otimes \mathscr{L})[[h]]$ the product integrals $\mathscr{A} \vec{\prod}(1+d l[h])$ and $\mathscr{A} \overleftarrow{\prod}(1+d l[h])$ are the unique elements $Y[h]$ and $Z[h]$ of $(\mathscr{A} \otimes \mathscr{T}(\mathscr{L}))[[h]]$ which satisfy the left and right differential equations

$$
(\mathrm{id} \mathscr{A} \otimes \vec{d}) Y[h]=Y^{12}[h] l^{13}[h],(\operatorname{id} \mathscr{A} \otimes \overleftarrow{d}) Z[h]=Z^{13}[h] l^{12}[h]
$$

together with the initial conditions

$$
(\text { id } \mathscr{A} \otimes \varepsilon) Y[h]=1_{\mathscr{A}},(\text { id } \mathscr{A} \otimes \varepsilon) Z[h]=1_{\mathscr{A}}
$$

where $\varepsilon$ is the counit of the Itô Hopf algebra $\mathscr{T}(\mathscr{L})$. Equivalently

$$
\begin{aligned}
& Y[h]=1_{\mathscr{A} \otimes \mathscr{T}(\mathscr{L})}+\sum_{n=1}^{\infty}\left(l^{12}[h] l^{13}[h] \cdots l^{1 n+1}[h]\right), \\
& Z[h]=1_{\mathscr{A} \otimes \mathscr{T}(\mathscr{L})}+\sum_{n=1}^{\infty}\left(l^{1 n+1}[h] l^{1 n}[h] \cdots l^{12}[h]\right),
\end{aligned}
$$

rearranged as formal power series with coefficients in $\mathscr{A} \otimes \mathscr{T}(\mathscr{L})$. Product integrals $\vec{\prod} \mathscr{A}(1+d m[h])$ and $\overleftarrow{\prod} \mathscr{A}(1+d m[h])$, where now $m[h] \in h(\mathscr{L} \otimes \mathscr{A})[[h]]$, are defined analogously in $(\mathscr{T}(\mathscr{L}) \otimes \mathscr{A})[[h]]$. If $\mathscr{A}$ is not necessarily unital, the decapitated

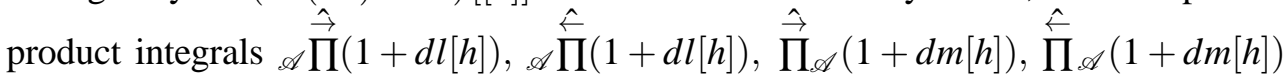
are defined by omitting the initial unit term from the expansion $[8]$ and its analogues. Thus $\hat{\overleftarrow{\Pi}}_{\mathscr{L}}(1+d r[h])$ and $\hat{\vec{L}}(1+d r[h])$ are defined as elements of $h(\mathscr{T}(\mathscr{L}) \otimes \mathscr{L})[[h]]$ and $h(\mathscr{L} \otimes \mathscr{T}(\mathscr{L}))[[h]]$ respectively, so that $\mathscr{T}(\mathscr{L}) \vec{\Pi}\left(1+d\left(\hat{\bigsqcup}_{\mathscr{L}}(1+d r[h])\right)\right.$ and $\overleftarrow{\Pi} \mathscr{T}(\mathscr{L})(1+d(\mathscr{\vec { \Pi }}(1+d r[h]))$ are defined as elements of $(\mathscr{T}(\mathscr{L}) \otimes \mathscr{T}(\mathscr{L}))[[h]]$. It is shown in [6] that these two iterated products are in fact equal. The double product integral $\stackrel{\leftarrow}{\Pi}(1+d r[h])$ is defined analogously by reversing the directions of all arrows.

Below we shall use the fact, proved in [6], that $\stackrel{\leftarrow \leftarrow}{\prod}(1+d r[h])$ and $\stackrel{\leftarrow}{\prod}(1+d r[h])$ are both of form

$$
1_{\mathscr{T}(\mathscr{L}) \otimes \mathscr{T}(\mathscr{L})}+r[h]+\beta[h]
$$

where $\beta[h]=\sum \beta_{m, n}[h]$ in $(\mathscr{T}(\mathscr{L}) \otimes \mathscr{T}(\mathscr{L}))[[h]]=\left(\bigoplus_{m, n=0}^{\infty}\left(\otimes^{m} \mathscr{L}\right) \otimes\left(\otimes^{n} \mathscr{L}\right)\right)[[h]]$ has nonzero homogeneous components $\beta_{m, n}[h]$ only if both $m$ and $n$ are nonzero and at least one exceeds 1 . 


\section{Properties of $\Delta$.}

In [6] it is shown that the element $R[h]=\vec{\prod}(1+d r[h])$ of $(\mathscr{T}(\mathscr{L}) \otimes \mathscr{T}(\mathscr{L}))[[h]]$ satisfies the quasitriangularity identities (1) and consequently, for arbitrary $m, n=$ $1,2, \ldots$

$$
\left(\Delta^{(m)} \otimes \Delta^{(n)}\right) R[h]=\prod_{(j, k) \in \mathbb{N}_{m} \times \mathbb{N}_{n}} R^{j, m+n+1-k}[h]
$$

in $\left(\left(\bigotimes^{m} \mathscr{T}(\mathscr{L})\right) \otimes\left(\bigotimes^{n} \mathscr{T}(\mathscr{L})\right)\right)[[h]]$. Here the notation is as follows. For $m=$ $1,2, \ldots, \mathbb{N}_{m}$ denotes the ordered set $(1,2, \ldots, m)$, and $\Delta^{(m)}: \mathscr{T}(\mathscr{L}) \rightarrow \bigotimes^{m} \mathscr{T}(\mathscr{L})$ is the $m$-fold coproduct which may be defined inductively by

$$
\Delta^{(1)}=\operatorname{id} \mathscr{T}(\mathscr{L}), \Delta^{(m)}=\left(\Delta \otimes \operatorname{id}_{\otimes^{m-1} \mathscr{T}(\mathscr{L})}\right) \circ \Delta^{(m-1)} .
$$

In particular $\Delta^{(2)}=\Delta . \stackrel{\leftarrow}{\prod}_{(j, k) \in \mathbb{N}_{m} \times \mathbb{N}_{n}} R^{j, m+n+1-k}[h]$ is the element of $\left(\left(\otimes^{m} \mathscr{T}(\mathscr{L})\right) \otimes\left(\bigotimes^{n} \mathscr{T}(\mathscr{L})\right)\right)[[h]]$ defined by by the equivalent prescriptions

$$
\prod_{(j, k) \in \mathbb{N}_{m} \times \mathbb{N}_{n}} R^{j, m+n+1-k}[h]=\prod_{j=1}^{m}\left\{\prod_{k=1}^{n} R^{j, m+n+1-k}[h]\right\}=\prod_{k=1}^{n}\left\{\prod_{j=1}^{m} R^{j, m+n+1-k}[h]\right\} .
$$

From the defining property of $\Delta$,

$$
\Delta\left(L_{1} \otimes L_{2} \otimes \cdots \otimes L_{m}\right)=\sum_{j=0}^{m}\left(L_{1} \otimes L_{2} \otimes \cdots \otimes L_{j}\right) \otimes\left(L_{j+1} \otimes L_{j+2} \otimes \cdots \otimes L_{m}\right)
$$

for arbitrary $L_{1}, L_{2}, \cdots, L_{m} \in \mathscr{L}$, we deduce that for the $m$ th order coproduct,

$$
\begin{aligned}
& \Delta^{(m)}\left(L_{1} \otimes L_{2} \otimes \cdots \otimes L_{m}\right) \\
& =\sum_{0 \leq j_{1} \leq j_{2} \leq \cdots \leq j_{m-1} \leq m}\left(L_{1} \otimes L_{2} \otimes \cdots \otimes L_{j_{1}}\right) \otimes \cdots \otimes\left(L_{j_{m-1}+1} \otimes L_{j_{m-1}+2} \otimes \cdots \otimes L_{m}\right) \\
& =L_{1} \otimes L_{2} \otimes \cdots \otimes L_{m}+\beta
\end{aligned}
$$

where $L_{1} \otimes L_{2} \otimes \cdots \otimes L_{m}$ is regarded as an element of $\bigotimes^{m} \mathscr{T}(\mathscr{L})=\bigotimes^{m}\left(\oplus_{n=0}^{\infty}\left(\bigotimes^{n} \mathscr{L}\right)\right)$ and the remainder $\beta$ consists of a sum of elements of each of which is of rank $>1$ in at least one copy of $\oplus_{n=0}^{\infty}\left(\otimes^{n} \mathscr{L}\right)$. Thus, by linearity, for general $\alpha=$ $\left(\alpha_{0}, \alpha_{1}, \alpha_{2}, \ldots\right) \in \mathscr{T}(\mathscr{L})$

$$
\left.\alpha_{m}=\left(\Delta^{(m)}(\alpha)\right)_{(1,1, \ldots, 1}^{(m)},{ }_{1}\right)
$$


in $\bigotimes^{m} \mathscr{T}(\mathscr{L})=\bigotimes^{m}\left(\oplus_{n=0}^{\infty}\left(\bigotimes^{n} \mathscr{L}\right)\right)$. (12) also holds when $m=0$ if $\Delta^{(0)}$ is defined to be the counit in $\mathscr{T}(\mathscr{L})$.

Now consider the products $R^{1,2}[h] R^{1,3}[h] R^{2,3}[h]$ and $R^{2,3}[h] R^{1,3}[h] R^{1,2}[h]$ in

$$
(\mathscr{T}(\mathscr{L}) \otimes \mathscr{T}(\mathscr{L}) \otimes \mathscr{T}(\mathscr{L}))[[h]]=\left(\bigoplus_{m, n, p=0}^{\infty}\left(\otimes^{m} \mathscr{L}\right) \otimes\left(\otimes^{n} \mathscr{L}\right) \otimes\left(\otimes^{p} \mathscr{L}\right)\right)[[h]]
$$

and denote by $\gamma_{m, n, p}[h], \tilde{\gamma}_{m, n, p}[h]$ their components of joint rank $(m, n, p)$. If any of the nonnegative integers $m, n, p$ is zero then $\gamma_{m, n, p}[h]$ and $\tilde{\gamma}_{m, n, p}[h]$ are formed entirely from only one of the three terms $R^{1,2}[h], R^{1,3}[h]$ and $R^{2,3}[h]$ and so must be equal. Assume that $(m, n, p) \in \mathbb{N}^{3}$. Then $\gamma_{m, n, p}[h]$ can be characterised as the component of $\left(\Delta^{(m)} \otimes \Delta^{(n)} \otimes \Delta^{(p)}\right)\left(R^{12}[h] R^{13}[h] R^{23}[h]\right)$ in $(\mathscr{T}(\mathscr{L}) \otimes \mathscr{T}(\mathscr{L}) \otimes \mathscr{T}(\mathscr{L}))[[h]]$ of joint rank $((1,1, \ldots, \stackrel{(m)}{1}),(1,1, \ldots, \stackrel{(n)}{1}),(1,1, \ldots, \stackrel{(p)}{1}))$. By $(10)$, and the multiplicativity of the iterated coproducts,

$$
\begin{aligned}
& \left(\Delta^{(m)} \otimes \Delta^{(n)} \otimes \Delta^{(p)}\right)\left(R^{1,2}[h] R^{1,3}[h] R^{2,3}[h]\right) \\
= & \prod_{(j, k) \in \mathbb{N}_{m} \times \mathbb{N}_{n}} R^{j, m+n+1-k}[h] \prod_{(j, l) \in \mathbb{N}_{m} \times \mathbb{N}_{p}} R^{j, m+n+p+1-l}[h] \prod_{(k, l) \in \mathbb{N}_{n} \times \mathbb{N}_{p}} R^{m+k, m+n+p+1-l}[h] .
\end{aligned}
$$

Thus $\gamma_{m, n, p}[h]$ is the component of the same joint rank in this expression. By (9) this is the component of the same joint rank in

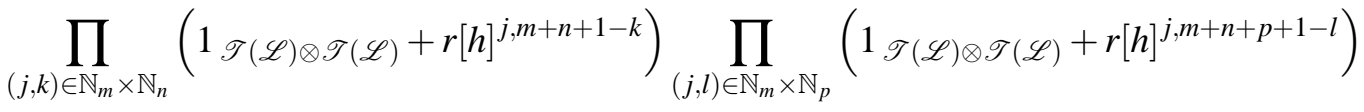

$$
\begin{aligned}
& \prod_{(k, l) \in \mathbb{N}_{n} \times \mathbb{N}_{p}}\left(1_{\mathscr{T}(\mathscr{L}) \otimes \mathscr{T}(\mathscr{L})}+r[h]^{m+k, m+n+p+1-l}\right)
\end{aligned}
$$

since higher order terms than $r[h]$ in the expansion (9) can only contribute to components in $\mathscr{T}(\mathscr{L}) \otimes \mathscr{T}(\mathscr{L})=\bigoplus_{m, n=0}^{\infty}\left(\otimes^{m} \mathscr{L}\right) \otimes\left(\otimes^{n} \mathscr{L}\right)$ at least one of whose ranks exceeds 1 . By expressing $\tilde{\gamma}_{m, n, p}[h]$ similarly as the component of the same joint rank in

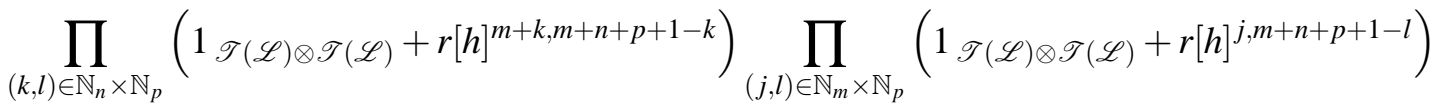

$$
\begin{aligned}
& \prod_{(k, l) \in \mathbb{N}_{n} \times \mathbb{N}_{p}}\left(1_{\mathscr{T}(\mathscr{L}) \otimes \mathscr{T}(\mathscr{L})}+r[h]^{j, m+n+1-l}\right)
\end{aligned}
$$

we complete the proof of the following.

Theorem 3 A necessary and sufficient condition that $R[h]=\stackrel{\leftarrow}{\prod}(1+d r[h])$ satisfy the quantum Yang-Baxter equation is that, for all triples $(m, n, p)$ of natural numbers, the components of joint rank $\left(\left(1,1, \ldots,{ }_{1}^{1}\right),\left(1,1, \ldots,,_{1}^{(n)}\right),\left(1,1, \ldots, 1^{1}\right)\right)$ in the products (13) and (14) are equal. 


\section{Proof of Theorem 1.}

From Theorem 3 we deduce that a necessary and sufficient condition for $R[h]=\vec{\prod}$ $(1+d r[h])$ to satisfy the quantum Yang-Baxter equation is that, for all $(m, n, p) \in \mathbb{N}^{3}$,

$$
\begin{aligned}
& \prod_{(j, k) \in \mathbb{N}_{m} \times \mathbb{N}_{n}} \rho^{j, m+n+1-k} \prod_{(j, l) \in \mathbb{N}_{m} \times \mathbb{N}_{p}} \rho^{j, m+n+p+1-l} \prod_{(k, l) \in \mathbb{N}_{n} \times \mathbb{N}_{p}} \rho^{m+k, m+n+p+1-l} \\
= & \prod_{(k, l) \in \mathbb{N}_{n} \times \mathbb{N}_{p}} \rho^{m+k, m+n+p+1-l} \prod_{(j, l) \in \mathbb{N}_{m} \times \mathbb{N}_{p}} \rho^{j, m+n+p+1-l} \prod_{(j, k) \in \mathbb{N}_{m} \times \mathbb{N}_{n}} \rho^{j, m+n+1}(\mathrm{k} 5)
\end{aligned}
$$

in the associative algebra $\left(\left(\otimes^{m} \mathscr{L}^{\prime}\right) \otimes\left(\otimes^{n} \mathscr{L}^{\prime}\right) \otimes\left(\otimes^{p} \mathscr{L}^{\prime}\right)\right)[[h]]$, where $\rho=1+r[h]$ and $h$ is suppressed for brevity. Indeed it is precisely these "pure Itô" terms which can contribute to the components of joint rank $\left((1,1, \ldots, \stackrel{(m)}{1}),(1,1, \ldots, \stackrel{(n)}{1}),\left(1,1, \ldots,{ }_{1}^{1}\right)\right)$ of the multitensors (13) and (14).

Taking $m=n=p=1$ we find that it is necessary that

$$
\rho^{1,2} \rho^{1,3} \rho^{2,3}=\rho^{2,3} \rho^{1,3} \rho^{1,2}
$$

from which we deduce (3) by expanding and cancelling the unit on each side.

To prove the sufficiency of (3), or equivalently of (16), we must show that it implies (15) for arbitrary $(m, n, p) \in \mathbb{N}^{3}$. First we prove the case when $m=n=1$ by induction on $p$; when $p=1$ this is just (16). With $m=n=1$ the left hand side of (15) becomes

$$
\begin{aligned}
& \rho^{1,2}\left\{\rho^{1, p+2} \rho^{1, p+1} \cdots \rho^{1,3}\right\}\left\{\rho^{2, p+2} \rho^{2, p+1} \cdots \rho^{2,3}\right\} \\
= & \left(\rho^{1,2} \rho^{1, p+2} \rho^{2, p+2}\right)\left\{\rho^{1, p+1} \rho^{1, p} \cdots \rho^{1,3}\right\}\left\{\rho^{2, p+1} \rho^{2, p} \cdots \rho^{2,3}\right\} \\
= & \rho^{2, p+2} \rho^{1, p+2} \rho^{1,2}\left\{\rho^{1, p+1} \rho^{1, p} \cdots \rho^{1,3}\right\}\left\{\rho^{2, p+1} \rho^{2, p} \cdots \rho^{2,3}\right\}
\end{aligned}
$$

using commutativity of $\rho^{2, p+2}$ with all but the first element of $\left\{\rho^{1, p+2} \rho^{1, p+1} \cdots \rho^{1,3}\right\}$ and (16). By the inductive hypothesis this is

$$
\begin{aligned}
& \rho^{2, p+2} \rho^{1, p+2}\left\{\rho^{2, p+1} \rho^{2, p} \cdots \rho^{2,3}\right\}\left\{\rho^{1, p+1} \rho^{1, p} \cdots \rho^{1,3}\right\} \rho^{1,2} \\
= & \left\{\rho^{2, p+2} \rho^{2, p+1} \cdots \rho^{2,3}\right\}\left\{\rho^{1, p+2} \rho^{1, p+1} \cdots \rho^{1,3}\right\} \rho^{1,2}
\end{aligned}
$$

which is the right hand side.

Next we prove the case $n=1$ by induction on $m$; when $m=1$ this becomes the case just proved. We have to show that, for arbitrary $m$ and $p$,

$$
\begin{aligned}
& \prod_{j=1}^{m} \rho^{j, m+1} \prod_{(j, l) \in \mathbb{N}_{m} \times \mathbb{N}_{p}} \rho^{j, m+2+p-l} \prod_{l=1}^{p} \rho^{m+1, m+p+2-l} \\
= & \prod_{l=1}^{p} \rho^{m+1, m+p+2-l} \prod_{(j, l) \in \mathbb{N}_{m} \times \mathbb{N}_{p}} \rho^{j, m+2+p-l} \prod_{j=1}^{m} \rho^{j, m+1}
\end{aligned}
$$


Using the first definition in 11 to define the double product and extracting the last internal product to the right, we express the left hand side as

$$
\begin{aligned}
& \prod_{j=1}^{m} \rho^{j, m+1} \prod_{(j, l) \in \mathbb{N}_{m-1} \times \mathbb{N}_{p}} \rho^{j, m+2+p-l} \prod_{l=1}^{p} \rho^{m, m+p+2-l} \prod_{l=1}^{p} \rho^{m+1, m+p+2-l} \\
= & \prod_{j=1}^{m-1} \rho^{j, m+1} \prod_{(j, l) \in \mathbb{N}_{m-1} \times \mathbb{N}_{p}} \rho^{j, m+2+p-l}\left(\rho^{m, m+1} \rho^{m, m+p+1} \rho^{m+1, m+p+1}\right) \\
& \rho^{m, m+p} \rho^{m, m+p-1} \cdots \rho^{m, m+2} \rho^{m+1, m+p} \rho^{m+1, m+p-1} \cdots \rho^{m+1, m+2}
\end{aligned}
$$

using the commutativity of $\rho^{m, m+1}$ with all remaining elements of the double product, and of $\rho^{m+1, m+p+1}$ with all but the first element of the extracted internal product. Reversing the bracketed Yang-Baxter triple, the terms after $\prod_{j=1}^{m-1} \rho^{j, m+1} \prod_{(j, l) \in \mathbb{N}_{m-1} \times \mathbb{N}_{p}} \rho^{j, m+2+p-l}$ become

$$
\begin{aligned}
& \left(\rho^{m+1, m+p+1} \rho^{m, m+p+1} \rho^{m, m+1}\right) \rho^{m, m+p} \rho^{m, m+p-1} \cdots \rho^{m, m+2} \\
& \rho^{m+1, m+p} \rho^{m+1, m+p-1} \cdots \rho^{m+1, m+2} \\
= & \rho^{m+1, m+p+1} \rho^{m, m+p+1}\left(\rho^{m, m+1} \rho^{m, m+p} \rho^{m+1, m+p}\right) \rho^{m, m+p-1} \rho^{m, m+p-2} \cdots \rho^{m, m+2} \\
& \rho^{m+1, m+p-1} \rho^{m+1, m+p-2} \cdots \rho^{m+1, m+2} \\
= & \rho^{m+1, m+p+1} \rho^{m, m+p+1}\left(\rho^{m+1, m+p} \rho^{m, m+p} \rho^{m, m+1}\right) \rho^{m, m+p-1} \rho^{m, m+p-2} \cdots \rho^{m, m+2} \\
& \rho^{m+1, m+p-1} \rho^{m+1, m+p-2} \cdots \rho^{m+1, m+2} \\
= & \rho^{m+1, m+p+1} \rho^{m, m+p+1} \rho^{m+1, m+p} \rho^{m, m+p}\left(\rho^{m, m+1} \rho^{m, m+p-1} \rho^{m+1, m+p-1}\right) \\
& \rho^{m, m+p-2} \cdots \rho^{m, m+2} \rho^{m+1, m+p-2} \rho^{m+1, m+p-3} \cdots \rho^{m+1, m+2} \\
= & \rho^{m+1, m+p+1} \rho^{m, m+p+1} \rho^{m+1, m+p} \rho^{m, m+p}\left(\rho^{m+1, m+p-1} \rho^{m, m+p-1} \rho^{m, m+1}\right) \\
& \rho^{m, m+p-2} \cdots \rho^{m, m+2} \rho^{m+1, m+p-2} \rho^{m+1, m+p-3} \cdots \rho^{m+1, m+2} \\
= & \cdots \\
= & \rho^{m+1, m+p+1} \rho^{m, m+p+1} \rho^{m+1, m+p} \rho^{m, m+p} \rho^{m+1, m+p-1} \rho^{m, m+p-1} \ldots \\
& \rho^{m+1, m+2} \rho^{m, m+2} \rho^{m, m+1} \\
= & \rho^{m+1, m+p+1} \rho^{m+1, m+p} \rho^{m+1, m+p-1} \cdots \rho^{m+1, m+2} \rho^{m, m+p+1} \rho^{m, m+p} \rho^{m, m+p-1} \ldots \\
& \rho^{m, m+2} \rho^{m, m+1} .
\end{aligned}
$$

Restoring the omitted initial terms $\prod_{j=1}^{m-1} \rho^{j, m+1} \prod_{(j, l) \in \mathbb{N}_{m-1} \times \mathbb{N}_{p}} \rho^{j, m+2+p-l}$ we obtain

$$
\prod_{j=1}^{m-1} \rho^{j, m+1}\left(\prod_{(j, l) \in \mathbb{N}_{m-1} \times \mathbb{N}_{p}} \rho^{j, m+2+p-l}\right) \prod_{l=1}^{p} \rho^{m+1, m+p+2-l} \prod_{l=1}^{p} \rho^{m, m+p+2-l} \rho^{m, m+1} .
$$

Applying the inductive hypothesis to the first three products, this becomes

$$
\prod_{l=1}^{p} \rho^{m+1, m+p+2-l}\left(\prod_{(j, l) \in \mathbb{N}_{m-1} \times \mathbb{N}_{p}} \rho^{j, m+2+p-l}\right) \prod_{j=1}^{m-1} \rho^{j, m+1} \prod_{l=1}^{p} \rho^{m, m+p+2-l} \rho^{m, m+1}
$$




$$
\begin{aligned}
& =\prod_{l=1}^{p} \rho^{m+1, m+p+2-l}\left(\prod_{(j, l) \in \mathbb{N}_{m-1} \times \mathbb{N}_{p}} \rho^{j, m+2+p-l}\right) \prod_{l=1}^{p} \rho^{m, m+p+2-l} \prod_{j=1}^{m} \rho^{j, m+1} \\
& =\prod_{l=1}^{p} \rho^{m+1, m+p+2-l}\left(\prod_{(j, l) \in \mathbb{N}_{m} \times \mathbb{N}_{p}} \rho^{j, m+2+p-l}\right) \prod_{j=1}^{m} \rho^{j, m+1}
\end{aligned}
$$

as required, where the last internal product of the original iterated product is restored again using the first definition in 111 to define the corresponding double product.

Finally we prove the general case by induction on $n$; when $n=1$ this is the case just proved.

We shall evaluate the first and last double products on the left hand side of (15) using respectively the second and first definitions of 111 . Detaching the last internal product from the first double product to the right and the first internal product from the third double product to the left, we obtain

$$
\begin{aligned}
& \left(\prod_{k=1}^{n-1}\left\{\prod_{j=1}^{m} \rho^{j, m+n+1-k}\right\}\right) \prod_{j=1}^{m} \rho^{j, m+1} \prod_{(j, l) \in \mathbb{N}_{m} \times \mathbb{N}_{p}} \rho^{j, m+n+p+1-l} \prod_{l=1}^{p} \rho^{m+1, m+n+p+1-l} \\
& \left(\prod_{k=2}^{n}\left\{\prod_{l=1}^{p} \rho^{m+k, m+n+p+1-l}\right\}\right)
\end{aligned}
$$

The middle three products in this expression can be reversed by (17) to get

$$
\begin{aligned}
& \left(\prod_{k=1}^{n-1}\left\{\prod_{j=1}^{m} \rho^{j, m+n+1-k}\right\}\right) \prod_{l=1}^{p} \rho^{m+1, m+n+p+1-l} \prod_{(j, l) \in \mathbb{N}_{m} \times \mathbb{N}_{p}} \rho^{j, m+n+p+1-l} \prod_{j=1}^{m} \rho^{j, m+1} \\
& \left(\prod_{k=2}^{n}\left\{\prod_{l=1}^{p} \rho^{m+k, m+n+p+1-l}\right\}\right)
\end{aligned}
$$

Since the elements $\rho^{m+1, m+n+p}, \rho^{m+1, m+n+p-1}, \cdots, \rho^{m+1, m+n+1}$ commute with those of the first iterated product in (18) and the elements $\rho^{1, m+1}, \rho^{2, m+1}, \cdots, \rho^{m, m+1}$ commute with those of the final iterated product, this is equal to

$$
\begin{aligned}
& \prod_{l=1}^{p} \rho^{m+1, m+n+p+1-l}\left(\prod_{k=1}^{n-1}\left\{\prod_{j=1}^{m} \rho^{j, m+n+1-k}\right\}\right) \prod_{(j, l) \in \mathbb{N}_{m} \times \mathbb{N}_{p}} \rho^{j, m+n+p+1-l} \\
& \left(\prod_{k=2}^{n}\left\{\prod_{l=1}^{p} \rho^{m+k, m+n+p+1-l}\right\}\right) \prod_{j=1}^{m} \rho^{j, m+1}
\end{aligned}
$$

We now use the inductive hypothesis to reverse the middle three double products to get

$$
\prod_{l=1}^{p} \rho^{m+1, m+n+p+1-l}\left(\prod_{k=2}^{n}\left\{\prod_{l=1}^{p} \rho^{m+k, m+n+p+1-l}\right\}\right) \prod_{(j, l) \in \mathbb{N}_{m} \times \mathbb{N}_{p}} \rho^{j, m+n+p+1-l}
$$




$$
\left(\prod_{k=1}^{n-1}\left\{\prod_{j=1}^{m} \rho^{j, m+n+1-k}\right\}\right) \prod_{j=1}^{m} \rho^{j, m+1}
$$

Finally we reabsorb the first and last products into the iterated products, to obtain

$$
\begin{aligned}
& \left(\prod_{k=1}^{n}\left\{\prod_{l=1}^{p} \rho^{m+k, m+n+p+1-l}\right\}\right) \prod_{(j, l) \in \mathbb{N}_{m} \times \mathbb{N}_{p}} \rho^{j, m+n+p+1-l}\left(\prod_{k=1}^{n}\left\{\prod_{j=1}^{m} \rho^{j, m+n+1-k}\right\}\right) \\
= & \prod_{(k, l) \in \mathbb{N}_{n} \times \mathbb{N}_{p}} \rho^{m+k, m+n+p+1-l} \prod_{(j, l) \in \mathbb{N}_{m} \times \mathbb{N}_{p}} \rho^{j, m+n+p+1-l} \prod_{(j, k) \in \mathbb{N}_{m} \times \mathbb{N}_{n}} \rho^{j, m+n+1-k}
\end{aligned}
$$

as required.

\section{Proof of Theorem 2.}

Being of the form $1_{\mathscr{T}(\mathscr{L}) \otimes \mathscr{T}(\mathscr{L})}+o(h), R[h]=\vec{\prod}(1+d r[h])$ possesses a multiplicative inverse $R[h]^{-1}$ of the same form. By the multiplicativity of $\Delta$ and the quasitriangularity relation $(\Delta \otimes \mathrm{id} \mathscr{T}(\mathscr{L})) R[h]=R^{13}[h] R^{23}[h]$

$$
\begin{aligned}
1_{\mathscr{T}(\mathscr{L}) \otimes \mathscr{T}(\mathscr{L}) \otimes \mathscr{T}(\mathscr{L})} & =\left(\Delta \otimes \mathrm{id} \mathscr{T}(\mathscr{L}) 1_{\mathscr{T}(\mathscr{L}) \otimes \mathscr{T}(\mathscr{L})}=\left(\Delta \otimes \mathrm{id}_{\mathscr{T}(\mathscr{L})}\right)\left(R[h] R[h]^{-1}\right)\right. \\
& =R^{1,3}[h] R^{2,3}[h]\left(\Delta \otimes \mathrm{id}_{\mathscr{T}(\mathscr{L})}\right)\left(R[h]^{-1}\right) .
\end{aligned}
$$

On the other hand the inverse of the product $R^{1,3}[h] R^{2,3}[h]$ is the product of the inverses in the reverse order $\left(R^{2,3}[h]\right)^{-1}\left(R^{1,3}[h]\right)^{-1}$. By uniqueness of inverses it follows that

$$
\left(\Delta \otimes \operatorname{id}_{\mathscr{T}(\mathscr{L})}\right)\left(R[h]^{-1}\right)=\left(R^{2,3}[h]\right)^{-1}\left(R^{1,3}[h]\right)^{-1} .
$$

A similar argument shows that

$$
(\operatorname{id} \mathscr{T}(\mathscr{L}) \otimes \Delta)\left(R[h]^{-1}\right)=\left(R^{1,2}[h]\right)^{-1}\left(R^{1,3}[h]\right)^{-1} .
$$

Hence $R[h]^{-1}$ satisfies the reverse quasitriangularity relations. Since it is manifestly nonzero, by the characterisation theorem of [6] it must be of form $R[h]^{-1}=\overleftrightarrow{\prod}(1+$ $\left.d r^{\prime}[h]\right)$ for some $r^{\prime}[h] \in h(\mathscr{L} \otimes \mathscr{L})[[h]]$. By comparing terms of rank $\leq 1$ in each copy of $\mathscr{T}(\mathscr{L})$ in the identity $R[h] R[h]^{-1}=1$ we find that $r^{\prime}[h]$ is the quasiinverse of $r[h]$.

\section{An example.}

We consider the two-dimensional complex associative algebra $\mathscr{L}$ spanned by elements $L, K$ with multiplication $L K=L, K^{2}=K, L^{2}=K L=0$ which has the matrix representation

$$
L \mapsto\left[\begin{array}{ll}
0 & 1 \\
0 & 0
\end{array}\right], K \mapsto\left[\begin{array}{ll}
0 & 0 \\
0 & 1
\end{array}\right]
$$


and for which the commutator Lie algebra is the non-Abelian two-dimensional Lie algebra. Then $\mathscr{L} \wedge \mathscr{L}$ is spanned by the element $r_{1}=L \otimes K-K \otimes L . r_{1}$ satisfies the classical Yang-Baxter equation; it defines the unique coboundary Lie bialgebra structure [4] on this Lie algebra. Also $r_{1}^{2}=0$ in the associative algebra $\mathscr{L} \otimes \mathscr{L}$ and

$$
r_{1}^{1,2} r_{1}^{1,3} r_{1}^{2,3}=r_{1}^{2,3} r_{1}^{1,3} r_{1}^{1,2}=0
$$

in $\mathscr{L} \otimes \mathscr{L} \otimes \mathscr{L}$. It follows from (19) that the equations (6) can be satisfied by taking all $r_{N}$ with $N>1$ to be 0 . Thus by Theorem $1, R[h]=\stackrel{\leftarrow}{\prod}\left(1+h d r_{1}\right)$ satisfies the quantum Yang-Baxter equation. Since $r_{1}^{2}=0$, the quasiinverse of $h r_{1}$ in $h \mathscr{L}[[h]]$ is $-h r_{1}$ so that by Theorem 2 the inverse of $R[h]$ in $(\mathscr{T}(\mathscr{L}) \otimes \mathscr{T}(\mathscr{L}))[[h]]$ is $R[h]^{-1}=\overleftarrow{\prod}\left(1-h d r_{1}\right)$.

To find the actions $\Delta[h](L)$ and $\Delta[h](K)$ of the deformed coproduct $\Delta[h]$ on $L$ and $K$, we use the commutation relations in $\mathscr{L} \otimes \mathscr{L}$

$$
\left[r_{1}, L^{1}\right]=L \otimes L,\left[r_{1}, L^{2}\right]=-L \otimes L,\left[r_{1}, K^{1}\right]=L \otimes K,\left[r_{1}, K^{2}\right]=-K \otimes L .
$$

Then, by (12), to find the homogeneous component of joint rank $(m, n)$ of

$\Delta[h](L)=\vec{\prod}\left(1+h d r_{1}\right) \Delta(L) \overleftarrow{\prod}\left(1-h d r_{1}\right)=\vec{\prod}\left(1+h d r_{1}\right)\left(L^{1}+L^{2}\right) \overleftarrow{\prod}\left(1-h d r_{1}\right)$ in $(\mathscr{T}(\mathscr{L}) \otimes \mathscr{T}(\mathscr{L}))[[h]]$, we compute the homogeneous component of joint rank $(1,1, \ldots, \stackrel{(m)}{1}, 1,1, \ldots, \stackrel{(n)}{1})$ of

$$
\begin{aligned}
& \left(\Delta^{(m)} \otimes \Delta^{(n)}\right) \Delta[h](L) \\
& =\left(\Delta^{(m)} \otimes \Delta^{(n)}\right)\left(\vec{\prod}\left(1+h d r_{1}\right)\right)\left(\Delta^{(m)} \otimes \Delta^{(n)}\right)\left(L^{1}+L^{2}\right) \\
& \left(\Delta^{(m)} \otimes \Delta^{(n)}\right)\left(\stackrel{\leftarrow}{\prod}\left(1-h d r_{1}\right)\right) \\
& =\prod_{(j, k) \in \mathbb{N}_{m} \times \mathbb{N}_{n}}\left(\vec{\prod}\left(1+h d r_{1}\right)\right)^{j, m+n+1-k}\left(L^{1}+L^{2}+\cdots+L^{m+n}\right) \\
& \prod_{(j, k) \in \mathbb{N}_{m} \times \mathbb{N}_{n}}\left(\overleftarrow{\prod}\left(1-h d r_{1}\right)\right)^{m+1-j, m+k}
\end{aligned}
$$

in $\left(\otimes^{m} \mathscr{T}(\mathscr{L})\right) \otimes\left(\otimes^{n} \mathscr{T}(\mathscr{L})\right)$, or equivalently of the product in $\left(\otimes^{m} \mathscr{L}^{\prime}\right) \otimes\left(\otimes^{n} \mathscr{L}^{\prime}\right)$

$$
\begin{aligned}
& \prod_{(j, k) \in \mathbb{N}_{m} \times \mathbb{N}_{n}}\left(1+h r_{1}\right)^{j, m+n+1-k}\left(L^{1}+L^{2}+\cdots+L^{m+n}\right) \prod_{(j, k) \in \mathbb{N}_{m} \times \mathbb{N}_{n}}\left(1-h r_{1}\right)^{m+1-j, m+k} \\
= & L^{1}+L^{2}+\cdots+L^{m+n}
\end{aligned}
$$


since by starting from the right of the first double product, each term $\left(1+h d r_{1}\right)^{j, m+n+1-k}$ in turn commutes with $L^{j}+L^{m+n+1-k}$ by (20) and with all the other $L^{l}$ since they occupy disjoint spaces, and may thus be cancelled with the its inverse in the second double product. This is evidently zero unless $(m, n)=(1,0)$ or $(0,1)$. It follows that $\Delta[h](L)=L \otimes 1+1 \otimes L$.

Similarly, the $(m, n)$ th component of $\Delta[h](K)$ is the $(1,1, \ldots, \stackrel{(m)}{1}, 1,1, \ldots, \stackrel{(n)}{1})$ th component of

$$
\prod_{(j, k) \in \mathbb{N}_{m} \times \mathbb{N}_{n}}\left(1+h r_{1}\right)^{j, m+n+1-k}\left(K^{1}+K^{2}+\cdots+K^{m+n}\right) \prod_{(j, k) \in \mathbb{N}_{m} \times \mathbb{N}_{n}}\left(1-h r_{1}\right)^{m+1-j, m+k},
$$

whence, using [20], we find $(\Delta[h](K))_{0,0}=0,(\Delta[h](K))_{1,0}=(\Delta[h](K))_{0,1}=K$,

$$
\begin{aligned}
(\Delta[h](K))_{1,1}=\left(\left(1+h r_{1}\right)\left(K^{1}+K^{2}\right)\left(1-h r_{1}\right)\right)_{1,1}=h(L \otimes K-K \otimes L) \\
(\Delta[h](K))_{2,1}=\left(\left(1+h r_{1}\right)^{1,3}\left(1+h r_{1}\right)^{2,3}\left(K^{1}+K^{2}+K^{3}\right)\left(1-h r_{1}\right)^{2,3}\left(1-h r_{1}\right)^{1,3}\right)_{1,1,1} \\
=\left(\left(1+h r_{1}\right)^{1,3} h\left(L^{2} K^{3}-K^{2} L^{3}\right)\left(1-h r_{1}\right)^{1,3}\right)_{1,1,1} \\
=h^{2}(L \otimes K-K \otimes L) \otimes L
\end{aligned}
$$

and generally for $m \geq 2$

$$
(\Delta[h](K))_{m, 1}=(-h)^{m}\left(\otimes^{m-2}(L)\right) \otimes(L \otimes K-K \otimes L) \otimes L,
$$

while similarly, for $n \geq 2$

$$
(\Delta[h](K))_{1, n}=h^{n} L \otimes(L \otimes K-K \otimes L) \otimes\left(\otimes^{n-2}(L)\right) .
$$

Also, using (20) repeatedly and the fact that $h r_{1}^{1,4}$ commutes with $L^{1} K^{4}-K^{4} L^{1}=r_{1}^{1,4}$

$$
\begin{aligned}
(\Delta[h](K))_{2,2}= & \left(\left(1+h r_{1}\right)^{1,4}\left(1+h r_{1}\right)^{1,3}\left(1+h r_{1}\right)^{2,4}\left(1+h r_{1}\right)^{2,3}\left(K^{1}+K^{2}+K^{3}+K^{4}\right)\right. \\
& \left.\left(1-h r_{1}\right)^{2,3}\left(1-h r_{1}\right)^{2,4}\left(1-h r_{1}\right)^{1,3}\left(1-h r_{1}\right)^{1,4}\right)_{1,1,1,1} \\
= & \cdots=\left(1+h r_{1}\right)^{1,4} h^{3}\left(-K^{1} L^{2} L^{3} L^{4}+L^{1} L^{2} L^{3} K^{4}\right)\left(1-h r_{1}\right)=0
\end{aligned}
$$

whence also $(\Delta[h](K))_{m, n}=0$ whenever $m, n \geq 2$. Thus $\Delta[h](K)$ is completely determined. Note that the components of ranks $(m, 1)$ and $(1, n)$ for $m, n \geq 2$ do not belong to $(\mathscr{S}(\mathscr{L}) \otimes \mathscr{S}(\mathscr{L}))[[h]]$.

\section{References}

[1] B Enriquez, Quantization of Lie bialgebras and shuffle algebras of Lie algebras, Selecta Math. (N.S.) 7, 321-407 (2001). 
[2] B Enriquez, Comment on "Double product integrals and Enriquez quantisation of Lie bialgebras I: The quasitriangularity relations", [Hudson and Pulmannová, Journal of Mathematical Physics 45, 2090-2105 (2004)], Journal of Mathematical Physics 45, 2106-2107 (2004).

[3] P Etingof and D Kazhdan, Quantization of Lie bialgebras I, Selecta Math.2, 1-41 (1996), II, ibid 4, 213-231 (1998), III, ibid 4, 233-269 (1998).

[4] P Etingof and O Schiffman, Lectures on quantum groups, International Press (1998).

[5] R L Hudson, Calculus in enveloping algebras, Jour. London Math. Soc. (2) 65, 361-380 (2002).

[6] R L Hudson and S Pulmannová, Double product integrals and Enriquez quantisation of Lie bialgebras I: The quasitriangularity relations, Journal of Mathematical Physics 45, 2090-2105 (2004).

[7] R L Hudson and S Pulmannová, Symmetrized double quantum stochastic product integrals, Journal of Mathematical Physics 41, 8249-8262 (2000). 